\title{
Application of the Phase Recovery Algorithm Based on the Parallel Architecture in the Holographic Projection Technology
}

\author{
Guangzhi Wu, Mingxin Kou, Qian He \\ China Satellite Maritime Tracking and Control Department, Jiangyin, 214431, China
}

\begin{abstract}
Compared with conventional display technology, holographic projection technology has outstanding advantages. The phase retrieval algorithm is a key image processing algorithms in holographic projection technology. The constringency speed and precision of these algorithms need to be raised. The performance of the algorithms above are not satisfactory, sometimes they cannot even guarantee that the iterative process converges to the correct solutions. Then, this paper proposes a parallel architecture based on the phase recovery algorithm-Parallel Gerchberg-Saxton with Genetic Algorithm (PGSGA). Simulation has proved that the proposed algorithm PGSGA is better in convergence rate and the restoration quality by testing gray-scale image.
\end{abstract}

Keywords: Holography; ViHPS; Phase Retrieval Algorithm; Phase-Only Hologram; GS Algorithm.

\section{Introduction}

A Holographic laser projection technology has been growing concern as a new technology. It compared with traditional image technology has obvious advantages - extremely efficient and safe, and can only display an image in a specific location or point of view; it can not only realize the traditional display of all achieved advanced technical indicators, such as large-screen, high-resolution, digitization, and laser as a light source has a wavelength selectivity and high spectral brightness and other characteristics, and has a wide color gamut, high color saturation and so on. Holographic laser projection technology requires less parts and good imaging effect, which means that the entire volume of the projection system can be made small, convenient equipped to mobile devices.

\section{ViPS principle}

Holographic video projection system (ViHPS) is the use of pure phase hologram projection imaging system. The use of coherent light irradiation plane a pure phase hologram, diffracted light is projected onto the image plane (Image Plane), will form an image. Pure phase hologram using light diffraction imaging, pure phase holograms amplitude distribution is a fixed constant amplitude modulation because it does not light, the light transmitted or reflected almost completely full advantage of the phase-type hologram diffraction efficiency high characteristics, therefore, in theory, and the entire imaging process is almost no light loss. By LCoS device for phase modulation of the incident light, you can get a pure phase hologram. In addition, a two-dimensional hologram reconstructed image quality is not affected device defects, defective pixels or noise caused by the region will be dispersed over the entire image, thereby greatly reducing their impact on the overall image quality.

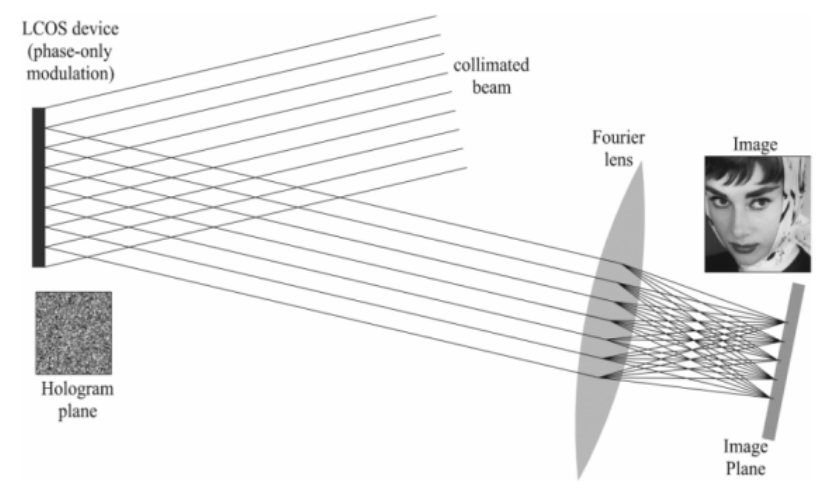

Figure 1 Video holographic projection system principle schematic diagram 
As shown, the same phase of the incident beam parallel beam, after reflection LCoS phase values of reflected light on the hologram plane different pixels to be changed, this completes the phase modulation of the light beam, the phase-modulated reflected light and then through a Fourier lens forming the final image. In the LCoS phase modulation process, the amplitude of the optical signal remains constant, the output can be phase modulated by LCoS device. As long as the appropriate phase of selecting a matrix, you can finally get a complete image on the image plane.

\section{Phase retrieval algorithm}

ViHPS in LCoS devices can complete phase-modulation hologram, thus reproduced a final image. But now most of the image display system are grayscale modulation approach, the input signal amplitude is gray matrix, the matrix phase is not ViHPS in need, so how according to the gray image amplitude information derived pure phase hologram this information has become a subject of urgent need to solve the problem. Such problems exist in many areas of physics, namely how to retrieve information easily measured intensity of the phase information is lost, the wave function --rebuilding phase recovery.

For electron microscopy techniques by measuring the strength of double recovery phase more successful and efficient algorithm is a set of GS 1971 Gerchberg and Saxton algorithm proposed, then, many scholars put forward a valid GS correction algorithm. In 1981, Gu origin and Yang Zhen and Dong Bizhen, who according to the general optical transformation theory formula proposed amplitude-phase general conversion system restoration under more general description of the way, and through strict mathematical derivation is given to determine the amplitude of a phase equation group, an iterative method that can handle a variety of amplitude-phase retrieval problems.

In the current phase retrieval problem solving algorithm system, GS algorithm is the most representative, and sometimes it can be quickly and get convergence error smaller result, the Fourier transform of the analytic situation, especially between the input and output for plane. Most of the phase retrieval algorithms are modifications GS algorithm.

Y-G propose any general theory of linear transformation phase recovery system, and successfully applied in a variety of conversion system, to solve various practical problems raised amplitude - phase retrieval problem can be divided into three categories: pure phase type, a pure amplitude, amplitude and phase hybrid, and gives the corresponding equations and efficient iterative algorithm. Yang - Gu phase retrieval algorithm is the second GS algorithm having a universal optimization algorithms, which iteration has two loop. In the case of calculating the same number of times, Yang - Gu phase retrieval algorithm faster than the GS algorithm is slow, but the convergence precision than the GS algorithm is high, it can be seen as GS algorithm promotion.

\section{Phase recovery algorithm based on parallel structure}

Yang - Gu phase retrieval algorithm which iteration has two loop, in the calculation of the number of times the same situation, Yang - Gu phase retrieval algorithm slower rate than GS, and therefore we consider accelerated algorithm based on the GS. Taking into account the convergence of the algorithm and GS Initial phase of selection, and therefore can be considered simultaneously in parallel operation of a plurality of the initial value, and constantly "sieve" the best way to accelerate the results of the GS algorithm. Taking into account the structure of the parallel computing features, here learn genetic algorithm [5] in the crossover and mutation operator for phase correction, so you can make a multi-phase corrected result as close as possible to the optimum result, the iterative results closer to the optimal solution, so as to further accelerate the optimization algorithm to GS. Based on this data processing thought, this paper based on parallel structure, genetic algorithm genetic, and mutation operator of GS to accelerate the phase correction phase recovery algorithm (ie PGSGA algorithm). The specific operation process is as follows:

Group $\mathrm{K}$ above formula uses the same arithmetic unit, the operation unit includes a two-dimensional inverse discrete Fourier transform $\left(\mathrm{FT}^{-1}\right)$, hologram phase calculating 
two-dimensional discrete Fourier transform (FT), and the phase image calculated error calculation, arithmetic total of five modules. Due to the above formula is a group K simultaneous operation, so the whole operation time and only count the same set of time, of course, the arithmetic processing resources consumed by the group $\mathrm{K}$ is $\mathrm{K}$ times as a group.

$$
\begin{aligned}
& H_{1}^{(n)}=F T^{-1}\left(G_{0} \bullet \exp \left(i \varphi_{1}^{(n)}\right)\right. \\
& \varphi_{1}^{(n)}=\operatorname{angle}\left(H_{1}^{(n)}\right) \\
& g_{1}^{(n)}=F T\left(H_{0} \sqsubset \exp \left(i \varphi_{1}^{(n)}\right)\right. \\
& \theta_{1}=\operatorname{angle}\left(g_{1}^{(n)}\right) \\
& \text { error }_{1}=R M S\left(\operatorname{abs}\left(g_{1}^{(n)}\right), G_{0}\right) \\
& H_{2}^{(n)}=F T^{-1}\left(G_{0} \sqsubset \exp \left(i \varphi_{2}^{(n)}\right)\right. \\
& \varphi_{2}^{(n)}=\operatorname{angle}\left(H_{2}^{(n)}\right) \\
& g_{2}^{(n)}=F T\left(H_{0} \sqsubset \exp \left(i \varphi_{2}^{(n)}\right)\right. \\
& \theta_{2}=\operatorname{angle}\left(g_{2}^{(n)}\right) \\
& \text { error }_{2}=R M S\left(a b s\left(g_{2}^{(n)}\right), G_{0}\right) \\
& H_{K}^{(n)}=F T^{-1}\left(G_{0} \sqsubset \exp \left(i \varphi_{K}^{(n)}\right)\right. \\
& \varphi_{K}^{(n)}=\operatorname{angle}\left(H_{K}^{(n)}\right) \\
& g_{K}^{(n)}=F T\left(H_{0} \sqsubset \exp \left(i \varphi_{K}^{(n)}\right)\right. \\
& \theta_{K}=\operatorname{angle}\left(g_{K}^{(n)}\right) \\
& \text { error }_{K}=R M S\left(a b s\left(g_{K}^{(n)}\right), G_{0}\right) \\
& {\left[\begin{array}{lllll}
\varphi_{1}^{(n+1)} & \varphi_{2}^{(n+1)} & \cdots \cdots & \varphi_{K}^{(n+1)} & \text { phase }_{0}
\end{array}\right]} \\
& \text { Phase }_{\text {Correction }}\left(\theta_{1} \quad \ldots . . . \theta_{K} \quad \text { error }_{1} \quad \ldots . . . \quad \text { error }_{K}\right)
\end{aligned}
$$

In the above formula, Phase_Correction processing module according to the respective error in front of $\mathrm{K}$ processing units, pick out a set of minimum error, and the phase data use crossover and mutation operators in some other parallel line should be amended to prevent premature convergence algorithm local minimum, but it can also accelerate the convergence speed.

\section{Phase Correction}

In the phase correction, using genetic algorithm crossover and mutation operator for the preceding phase correction. In the genetic algorithm, crossover and mutation operator role is to generate new chromosomes, change the search path. Referenced herein may be changed for the next iteration of the initial value, so that the next iteration, iteration result of stagnation in the local minimum probability becomes low, and thus have more chance of approaching the optimal solution, and thus achieve the purpose of accelerating. Set forth below crossover and mutation methods described herein taken.

First, the purpose of cross mutation prevents GS algorithm stagnation in local minimum; two GS can accelerate convergence to the optimum speed (by changing the initial iteration phase).

Pure phase hologram can be calculated by the above iterative method for pure phase holograms corresponding amplitude information (corresponding to a constant) can be directly calculated. Assuming pure phase hologram information corresponding amplitude value constants a, image intensity matrix $\mathrm{M}$, the matrix of size $\mathrm{m} * \mathrm{n}$, according Pasaiwaer theorem, there are:

$$
a=\frac{1}{m n} \sqrt{\sum_{i} \sum_{j}(M(i, j))^{2}}
$$

Since ViPS is the use of pure phase hologram image amplitude information, phase-only hologram is a fixed constant a.

\section{Simulation}

Matlab software simulation is GS, Fieup, PGSGA algorithm to discuss their performance parameters were compared and analyzed. 
$256 * 256$ resolution lena image GS, Fieup, PGSGA iterative algorithm 5,10,20,30 times and record the results of the iteration as follows:

The initial phase of iterative image matrix is set to zero. Here are three algorithms iterative convergence curve, the curve describes the restoration of the original image and the image of the mean square error with the number of iterations of the curve, as shown below:

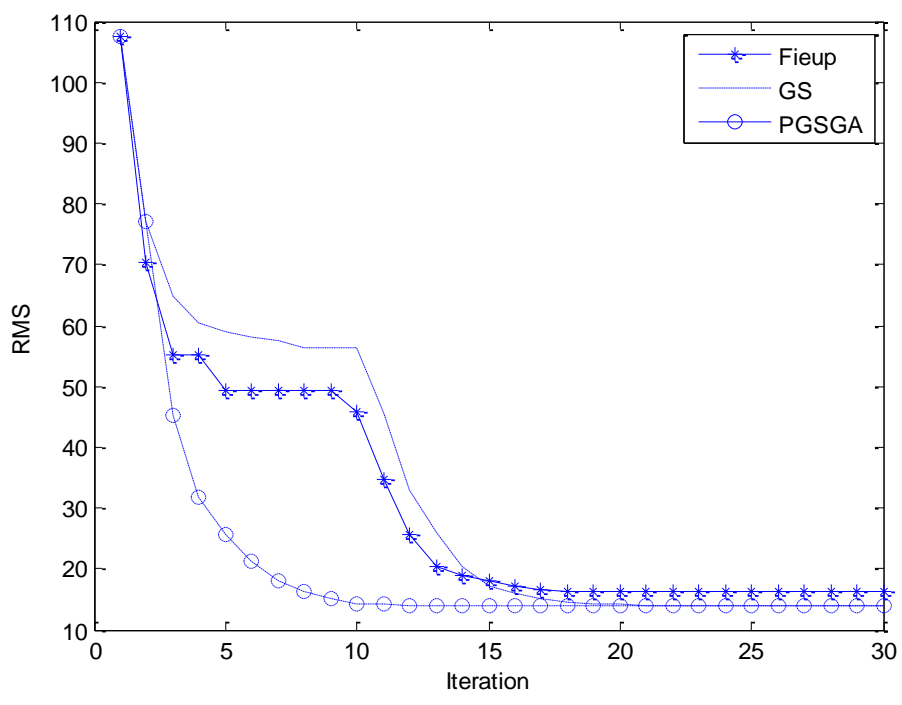

Figure 2 Change curve of mean square error with the number of iteration

Figure 2 curve describes the iterative convergence curve GS, Fieup, PGSGA algorithm. The first 15 iteration convergence result PGSGA also superior GS, Fieup. The fewer the number of iterations of the algorithm, hardware operating time will be relatively reduced.

Therefore PGSGA algorithms on performance parameters are more GS, Fieup algorithm is superior, 10 iterations RMS error should be reduced by more than $60 \%$, which means that the PGSGA implement algorithms in hardware, the number may be a few iterations to meet or exceed GS , Fieup algorithm more iterations iterative effect.

\section{Summary}

Based on research and analysis GS iterative algorithm proposed GS iterative algorithm parallel structure (PGSGA) based on the algorithm of computing parallel structure, drawing on genetic algorithm crossover and mutation operators thought iteration result is not ideal the part is corrected by changing the first phase of the next iteration of the initial value to accelerate convergence. The results showed that, PGSGA algorithm can calculate the pure phase hologram, recover the image quality and the mean square error is more GS, Fieup algorithm is superior. Through Matlab simulation software to verify the validity PGSGA algorithm, with the GS, Fieup algorithm is verified by comparing the superiority of the algorithm.

\section{References}

[1]. Gerchberg R W, Saxton W O. Phase determination from imagines and diffraction plane pictures in the election micro scope [J]. Optik, 2011, 34(2): 275-283.

[2]. A. Georgiou and W. A. Crossland. Image projection using phase-only holograms [J]. Photonic Conference Proceedings, 2013.

[3]. Dai H, Liu K X Y, Wang X and Liu J. Characteristics of LCOS phase-only spatial light modulator and its applications[J]. Opt. Commun. 2012, 238.

[4]. A. Georgiou,J. Christmas,J. Moore,A. Jeziorska-Chapman,A. Davey,N. Collings,and W. A Crossland. Liquid crystal over silicon device characteristics for holographic projection of high-definition television images [J]. Applied Optics. 2008, 26(47): 4793-4804. 